\title{
Concordância entre diagnósticos neuropatológicos premortem e postmortem em 90 pacientes com síndrome da imunodeficiência adquirida, submetidos à necrópsia em um hospital de ensino no Brasil
}

\author{
Ana Cristina Araújo Lemos da Silva', Blenda Sousa Carli Rodrigues², \\ Everton Nunes de Melo Moura ${ }^{3}$, Antonio Carlos Oliveira de Meneses 4 , \\ Adilha Misson Rua Micheletti ${ }^{4}$, Mario Leon Silva Vergara ${ }^{5}$, Sheila Jorge Adad 6 \\ Trabalho realizado na Universidade Federal do Triângulo Mineiro (UFTM) - MG.
}

\section{RESUMO}

Objetivo: Estudar as lesões anatomopatológicas no SNC, analisando se contribuíram para o óbito desses pacientes e avaliar a concordância entre os diagnósticos neuropatológicos premortem e postmortem. Método: Estudo retrospectivo com análise de 90 necrópsias sequenciais de SIDA, realizadas entre 1989 e 1996, além de busca de dados epidemiológicos/clínicos e comparação com a suspeita clínica para avaliar concordância entre diagnóstico pre e postmortem. Resultados: Idade média $34 \pm 11$ anos; $81,1 \%$ do sexo masculino; todos apresentavam alguma alteração no SNC, sendo relevantes para o óbito em 54 (60\%) casos. Apenas em 15 (27,8\%) desses 54 casos houve suspeita clínica da lesão. As lesões encontradas no SNC foram: toxoplasmose $(34,4 \%)$, criptococose $(12,2 \%)$, citomegalovirose $(4,4 \%)$, encefalite nodular microglial $(3,3 \%)$, meningite bacteriana (2,2\%), infecção pelo vírus JC (2,2\%), tuberculose/micobacteriose (2,2\%), histoplasmose $(1,1 \%)$ e paracoccidioidomicose $(1,1 \%)$. As alterações não relacionadas ao óbito (40\%) foram: gliose, fibrose meníngea focal, infarto antigo, calcificações e edema. Conclusão: Este estudo confirma que existem diversas alterações no SNC de pacientes com SIDA, sobretudo infecções oportunistas, frequentemente não suspeitadas clinicamente, evidenciando a importância da necrópsia. Esses achados sugerem que investigação minuciosa deveria ser feita no SNC de pacientes com SIDA, particularmente naqueles com diagnóstico tardio ou que não estão respondendo ao tratamento.

\section{PALAVRAS-CHAVE}

Sistema nervoso central/lesões, síndrome de imunodeficiência adquirida, autopsia.

\section{ABSTRACT}

Agreement between premortem e postmortem neuropathological diagnoses in 90 patients with acquired immune deficiency syndrome, submitted to autopsy at a teaching hospital in Brazil Objective: The aim was to study the anatomopathological lesions in CNS, analyzing if they have contributed to the death of those patients, and to evaluate the agreement between premortem and postmortem neuropathological diagnoses. Method: Retrospective study on 90 necropsies on patients with AIDS, performed between 1989 and 1996, besides having been performed the search for clinical and epidemiologycals datas, was done a comparison with the clinical suspicion, to analyze the concordance between premortem and postmortem diagnoses. Results: The patients'

1. Mestre em Patologia, professor-assistente, disciplina de Patologia Especial da Universidade Federal do Triângulo Mineiro (UFTM), MG, Brasil.

2. Acadêmico de Medicina, UFTM, bolsista de iniciação científica, Conselho Nacional de Desenvolvimento Científico e Tecnológico (CNPq), MG, Brasil.

3. Acadêmico de Medicina, UFTM, bolsista de iniciação científica, Fundação de Amparo à Pesquisa do Estado de Minas Gerais (Fapemig), MG, Brasil.

4. Doutor em Patologia, professor adjunto, disciplina de Patologia Especial, UFTM, MG, Brasil.

5. Doutor em Clínica Médica, professor adjunto, disciplina de Doenças Infecciosas e Parasitárias, UFTM, MG, Brasil.

6. Doutor em Patologia, professor-associado, disciplina de Patologia Especial, UFTM, MG, Brasil. 
mean age was $34 \pm 11$ years, $81.1 \%$ were male. CNS lesions were found in all of the cases. In 54 $(60 \%)$ cases, CNS lesions of relevance to the death were found, but there had only been clinical suspicion of such lesions in 15 (27.8\%) of them. The lesions consisted of toxoplasmosis (34.4\%), cryptococcosis (12.2\%), cytomegalovirosis (4.4\%), microglial nodular encephalitis (3.3\%), bacterial meningitis (2.2\%), JC virus infection (2.2\%), tuberculosis/mycobacteriosis (2.2\%), histoplasmosis $(1.1 \%)$ and paracoccidioidomycosis (1.1\%). The unrelated lesions with the death (40\%) were: gliosis, meningeal fibrosis, old infarct, calcifications and edema. Conclusion: This study confirms that several changes occurred in the CNS of patients with AIDS, especially opportunistic infections, that frequently are not suspected clinically. These findings suggest that detailed investigation should be conducted on CNS of patients with AIDS, particularly those who are not responding to treatment or with late diagnoses.

\section{KEYWORDS}

Central nervous system/injuries, acquired immunodeficiency syndrome, autopsy.

\section{Introdução}

O envolvimento do sistema nervoso central em pacientes com SIDA é muito frequente $e^{5,6,11} e$, por vezes, mais difícil de ser tratado, quando comparamos com as mesmas infecções extracerebrais. ${ }^{11,6} \mathrm{~A}$ infecção pelo vírus da imunodeficiência (HIV) é frequente no mundo inteiro e há, no Brasil, mais de 500 mil casos de SIDA. ${ }^{4}$ Entretanto, existem poucos estudos com grandes séries de necrópsias de SNC/SIDA tanto no Brasil ${ }^{6,16}$ quanto em outros países $2,5,8,10,11,14,15$. Ademais, muitos pacientes, pelo menos em países subdesenvolvidos, morrem sem diagnóstico das lesões no SNC. O objetivo deste estudo foi estudar as lesões do SNC analisando se contribuíram para o óbito desses pacientes e avaliar a concordância entre os diagnósticos premortem e postmortem.

\section{Métodos}

Estudo retrospectivo de 90 necrópsias consecutivas de pacientes HIV positivos com SIDA, de um total de 847 necrópsias, realizadas no período de janeiro de 1989 a dezembro de 1996, na Universidade Federal do Triângulo Mineiro (UFTM), Uberaba - MG. Todos os casos tinham confirmação sorológica da infecção por HIV.

Foram revistas lâminas com cortes histológicos de material fixado em formol e processado para inclusão em parafina do SNC (áreas de lesões macroscópicas e amostras de regiões macroscopicamente normais do córtex frontal, temporal, parietal e occipital, hipocampo, núcleo lenticular, ponte, medula e cerebelo). A avaliação inicial foi em cortes corados pela técnica de hematoxilina-eosina e, para investigação de agentes infecciosos e doenças desmielinizantes, foram utilizados Grocott, mucicarmim, Fite-Faraco, PAS, Luxol fast blue, além de imuno-histoquímica para confirmação microbiológica de citomegalovirose (CMV) e toxoplasmose.

Com os dados obtidos por meio da revisão microscópica, foi feita uma análise comparando-se a causa de óbito do paciente com as alterações encontradas no SNC, na tentativa de verificar se essas lesões tiveram participação direta na causa da morte ou se foram achados irrelevantes em relação ao óbito. A seguir, nos casos cujas lesões anatomopatológicas do SNC eram significativas, fez-se a comparação com a suspeita clínica, a fim de estabelecer a concordância pre e postmortem. Para cada caso, consideramos que houve concordância entre os diagnósticos clínicos premortem e os diagnósticos postmortem, quando ambos eram similares. Foi considerado discordante quando o diagnóstico neuropatológico postmortem foi diferente da hipótese clínica ou não foi estabelecida hipótese clínica neuropatológica premortem. Essa forma de classificar representa uma simplificação do proposto por Goodman et al. ${ }^{9}$, aproximando-se mais da prévia simplificação feita por Almeida et al. ${ }^{1}$ A razão pela qual agrupamos as discrepâncias classes I (doença importante que, se diagnosticada clinicamente, alteraria o prognóstico) e II (não diagnóstico de doença importante que, se diagnosticada clinicamente, não alteraria o prognóstico) deve-se ao fato de serem essas classificações usualmente aplicáveis à análise de todas as doenças presentes no indivíduo e não apenas às alterações neuropatológicas.

Os resultados foram expressos por análise descritiva colocando-se a frequência das principais alterações do SNC observadas à necrópsia, sua associação com a causa de óbito, assim como em relação à hipótese premortem.

Este estudo foi aprovado pelo Comitê de Ética e Pesquisa da Universidade Federal do Triângulo Mineiro em 27/11/2006 (número 831). 


\section{Resultados}

Houve no período estudado 92 necrópsias de pacientes HIV; entretanto, 2 casos foram excluídos desta análise por não apresentarem SIDA. Desses 90 casos que fizeram parte do estudo, $73(81,1 \%)$ eram do sexo masculino e 17 (18,9\%), do sexo feminino, com média de idade de $34 \pm 11$ anos, variando entre 18 e 67 anos.

Em todos os casos foram encontradas lesões no SNC, sendo relevantes para o óbito em 54 (60\%) dos 90 casos. Apenas em 15 (27,8\%) desses 54 casos houve suspeita clínica da lesão encontrada à necrópsia. As principais lesões foram infecções, em geral oportunistas, que ocorreram em 54 (60\%) dos 90 casos (Tabela 1). Nenhum caso de neoplasia no SNC foi encontrado neste período.

A lesão mais frequente foi neurotoxoplasmose (31 casos; $34,4 \%$ ), seguida pela neurocriptococose ( 11 casos; $12,2 \%$ ), citomegalovirose ( 4 casos; $4,4 \%$ ), encefalite nodular microglial (3 casos; $3,3 \%$ ), meningite bacteriana ( 2 casos; $2,2 \%$ ), infecção pelo vírus JC ( 2 casos; $2,2 \%$ ), tuberculose/micobacteriose (2 casos; $2,2 \%$ ), histoplasmose (1 caso; $1,1 \%$ ) e paracoccidioidomicose ( 1 caso; $1,1 \%)$. Em três $(3,3 \%)$ casos havia duas infecções concomitantes (toxoplasmose + meningite purulenta; toxoplasmose + citomegalovirose; paracoccidioidomicose + citomegalovirose).

Dentre os 36 (40\%) casos restantes havia um infarto antigo extenso e outro com diversos focos necrótico-calcificados por sequela de antiga toxoplasmose. Nos demais casos, as lesões não pareciam relevantes clinicamente (glioses focais, calcificações e fibroses meníngeas focais), ou eram edemas associados à septicemia e/ ou anóxia. Alterações pelo HIV, no SNC, não foram diagnosticadas.

\section{Discussão}

A média de idade dos pacientes estudados (34 anos) foi similar à encontrada em outros estudos brasileiros $\mathrm{s}^{3,7}$ e menor do que aquela verificada nas séries de países desenvolvidos (38 anos), provavelmente devido ao maior tempo de sobrevida dos pacientes nesses países, apesar de essa comparação estar sendo feita com estudos realizados em época semelhante, ou seja, anterior ao uso da terapia antirretroviral combinada.

Analisando as lesões encontradas no SNC, o percentual de infecções oportunistas (60\%) foi similar ao de Moskowitz et al. ${ }^{13}$ (50\%), maior do que o de Wainstein et al. ${ }^{16}$ (35\%) e de Lang et al. ${ }^{11}(40 \%)$ e menor do que o de Chimelli et al. ${ }^{6}(65,4 \%)$ e Jellinger et al. ${ }^{10}(67,4 \%)$. Essas diferenças talvez possam ser explicadas pela maior ou menor amostragem do SNC para microscopia em cada estudo.

Considerando o tipo de infecção oportunista, a mais prevalente neste estudo foi neurotoxoplasmose $(34,4 \%)$, similar ao encontrado por Chimelli et al. ${ }^{6}$

\begin{tabular}{|c|c|c|}
\hline Alterações & $\mathrm{n}$ & $\%$ \\
\hline Infecções oportunistas* & 54 & 60,0 \\
\hline \multicolumn{3}{|l|}{ Protozoário } \\
\hline Toxoplasmose & 31 & 34,4 \\
\hline \multicolumn{3}{|l|}{ Fungos } \\
\hline Criptococose & 11 & 12,2 \\
\hline Histoplasmose & 01 & 1,1 \\
\hline Paracoccidioidomicose & 01 & 1,1 \\
\hline \multicolumn{3}{|l|}{ Bactérias } \\
\hline Meningite purulenta & 02 & 2,2 \\
\hline Tuberculose/micobacteriose & 02 & 2,2 \\
\hline \multicolumn{3}{|l|}{ Vírus } \\
\hline Citomegalovírus & 04 & 4,4 \\
\hline Vírus JC (LMP) & 02 & 2,2 \\
\hline Encefalite nodular microglial & 03 & 3,3 \\
\hline $\begin{array}{l}\text { Outras lesões (edema, gliose, fibro } \\
\text { infarto antigo, calcificações...) }\end{array}$ & 36 & 40,0 \\
\hline
\end{tabular}

${ }^{*}$ Havia 3 (3,3\%) casos com 2 infecções concomitantes (toxoplasmose + meningite purulenta; toxoplasmose + citomegalovírus; citomegalovírus + paracoccidioidomicose); LMP - leucoencefalopatia multifocal progressiva. 
$(34,1 \%)$, Moskowitz et al. ${ }^{14}(30,8 \%)$ e maior do que o de Anders et al. ${ }^{2}(6,7 \%)$, Petito et al ${ }^{15}(10 \%)$ e Jellinger et $a l .^{10}(20,4 \%)$. Essas discordâncias podem ter relação com diferenças geográficas e socioeconômicas dos locais de estudo, além da maior ou menor amostragem do SNC.

A segunda infecção mais frequente foi criptococose, diagnosticada em 12,2\% dos casos, assim como no estudo de Anders et al. ${ }^{2}(12,4 \%)$, Wainstein et al. ${ }^{16}$ (12\%) e Chimelli et al. ${ }^{6}(13,5 \%)$ e bem mais frequente que no estudo de Moskowitz et al. ${ }^{14}(1,9 \%)$, Petito et al. ${ }^{15}$ (menor que $3 \%$ ), Lang et al. ${ }^{11}(4 \%)$, Funata et al. ${ }^{8}$ $(6,6 \%)$ e Jellinger et al. ${ }^{10}(4,4 \%)$. O elevado percentual de criptococose neste estudo e, possivelmente das outras séries brasileiras, talvez possa ser explicado pelo fato de muitos pacientes não fazerem acompanhamento clínico regular, a julgar pelo que verificamos em nossos casos por meio da revisão dos prontuários, não sendo, então, submetidos ao tratamento dessa infecção, que é facilmente diagnosticada pelo exame do liquor.

A alta frequência de toxoplasmose e criptococose em nosso meio deve-se, provavelmente, à ausência de tratamento devido a fatores socioeconômicos. ${ }^{6}$ A frequência de infecção por CMV foi baixa $(4,4 \%)$, se comparada com outras séries, ${ }^{2,9,10,15}$ que variaram de $4 \%$ a $26 \%$. Por outro lado, estudo brasileiro de Porto Alegre ${ }^{16}$ não diagnosticou nenhuma infecção por CMV em 138 casos de necrópsia.

Nossos pacientes apresentaram várias complicações por toxoplasmose e criptococose (que podem ser facilmente tratadas quando diagnosticadas corretamente) e provavelmente não sobreviveram tempo suficiente para desenvolver outros tipos de lesões, como infecções virais e neoplasias. Esta pode ser a razão pela qual não identificamos nenhum caso de neoplasia do SNC neste estudo, fato que também ocorreu na série de Waisntein et al. ${ }^{16}$

Por outro lado, considerando que a região em que foi realizado este estudo tem um passado recente de área endêmica para a doença de Chagas, seria de se esperar algum caso de reativação devido à imunodeficiência, o que não foi detectado nesta série de necrópsias. Estudo anterior comparativo entre meningoencefalite chagásica e toxoplasmótica, feito na região, detectou 2 casos necrópsiados de reativação de doença de Chagas, porém, ambos eram de Uberlândia. ${ }^{12}$

\section{Conclusão}

Este estudo confirma que existe o risco de diversas manifestações patológicas no SNC em pacientes com SIDA, sendo principalmente doenças infecciosas oportunistas na nossa região, frequentemente não suspeitadas clinicamente, evidenciando a importância da necrópsia para melhor conhecimento das alterações. Esses achados sugerem que investigação minuciosa deve ser feita no SNC em pacientes com SIDA, especialmente aqueles com diagnóstico tardio da infecção pelo HIV e consequentemente já muito imunocomprometidos e, também, naqueles que não estão respondendo ao tratamento.

\section{Agradecimento}

Apoio financeiro: Fundação de Amparo à Pesquisa do Estado de Minas Gerais (Fapemig - CDS 2029/05 e CDS-APQ 5583-4.01/07)

Bolsa de Produtividade em Pesquisa, Conselho Nacional de Desenvolvimento Científico e Tecnológico (CNPq) - 309174/2008-2.

\section{Referências}

1. de Almeida MC, Couto LA, da Silva LH, Carvalhal Sdos S. [Anatomo-clinical diagnosis correlation. Retrospective assessment of the clinical diagnosis in necropsies]. Rev Saude Publica. 1989;23(4):285-91.

2. Anders $\mathrm{KH}$, Guerra WF, Tomiyasu U, Verity MA, Vinters HV. The neuropathology of AIDS. UCLA experience and review. Am J Pathol. 1986;124(3):537-58.

3. Borges AS, Ferreira MS, Nishioka Sda A, Silvestre MT, Silva AM, Rocha A. Agreement between premortem and postmortem diagnoses in patients with acquired immunodeficiency syndrome observed at a Brazilian teaching hospital. Rev Inst Med Trop Sao Paulo. 1997;39(4):217-21.

4. Brasil, Ministério da saúde. Aids em números. AIDS no Brasil. DST - Aids. [Acesso em: 24 maio 2010]. Disponível em: <http://www.aids.gov.br/data/Pages/ LUMIS13F4BF21ITEMID0CE4429BFBAD4595A0 DC9FE9327EDC83PTBRIE.htm >

5. Budka H, Costanzi G, Cristina S, Lechi A, Parravicini C, Trabattoni $R$, et al. Brain pathology induced by infection with the human immunodeficiency virus (HIV). A histological, immunocytochemical, and electron microscopical study of 100 autopsy cases. Acta Neuropathol. 1987;75(2):185-98.

6. Chimelli L, Rosemberg S, Hahn MD, Lopes MB, Netto MB. Pathology of the central nervous system in patients infected with the human immunodeficiency virus (HIV): a report of 252 autopsy cases from Brazil. Neuropathol Appl Neurobiol. 1992;18(5):478-88.

7. Cury PM, Pulido CF, Furtado VM, da Palma FM. Autopsy findings in AIDS patients from a reference hospital in Brazil: analysis of 92 cases. Pathol Res Pract. 2003;199(12):811-4.

8. Funata N, Maeda Y, Koike M, Okeda R. Neuropathology of the central nervous system in acquired immune deficiency syndrome (AIDS) in Japan. With special reference to human immunodeficiency virus-induced encephalomyelopathies. Acta Pathol Jpn. 1991;41(3):206-11. 
9. Goldman L, Sayson R, Robbins S, Cohn LH, Bettmann M, Weisberg $\mathrm{M}$. The value of the autopsy in three medical eras. N Engl J Med. 1983;308(17):1000-5.

10. Jellinger KA, Setinek U, Drlicek M, Böhm G, Steurer A, Lintner $F$. Neuropathology and general autopsy findings in AIDS during the last 15 years. Acta Neuropathol. 2000;100(2):213-20.

11. Lang W, Miklossy J, Deruaz JP, Pizzolato GP, Probst A, Schaffner T, et al. Neuropathology of the acquired immune deficiency syndrome (AIDS): a report of 135 consecutive autopsy cases from Switzerland. Acta Neuropathol. 1989;77(4):379-90.

12. Lazo JE, Meneses AC, Rocha A, Frenkel JK, Marquez JO, Chapadeiro E, et al. [Toxoplasmic and chagasic meningoencephalitis in patients with human immunodeficiency virus infection: anatomopathologic and tomographic differential diagnosis]. Rev Soc Bras Med Trop. 1998;31(2):163-71.

13. Moskowitz L, Hensley GT, Chan JC, Adams K. Immediate causes of death in acquired immunodeficiency syndrome. Arch Pathol Lab Med. 1985;109(8):735-8.
14. Moskowitz LB, Hensley GT, Chan JC, Gregorios J, Conley FK. The neuropathology of acquired immune deficiency syndrome. Arch Pathol Lab Med. 1984;108(11):867-72.

15. Petito CK, Cho ES, Lemann W, Navia BA, Price RW. Neuropathology of acquired immunodeficiency syndrome (AIDS): an autopsy review. J Neuropathol Exp Neurol. 1986;45(6):635-46.

16. Wainstein MV, Ferreira L, Wolfenbuttel L, Golbspan L, Sprinz $\mathrm{E}, \mathrm{Kronfeld} \mathrm{M}$, et al. [The neuropathological findings in the acquired immunodeficiency syndrome (AIDS): a review of 138 cases]. Rev Soc Bras Med Trop. 1992;25(2):95-9.

\section{Endereço para correspondência}

Ana Cristina Araújo Lemos da Silva

Disciplina de Patologia Especial

Av. Getúlio Guaritá, 130

38025-440 - Uberaba, MG, Brasil

Telefone: (34) 3318-5152

Email: anacals@gmail.com 